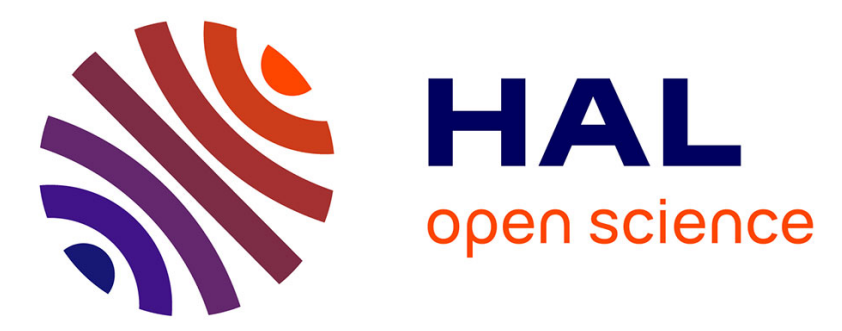

\title{
Joint State and Dynamics Estimation With High-Gain Observers and Gaussian Process Models
}

\author{
Mona Buisson-Fenet, Valery Morgenthaler, Sebastian Trimpe, Florent Di
}

Meglio

\section{- To cite this version:}

Mona Buisson-Fenet, Valery Morgenthaler, Sebastian Trimpe, Florent Di Meglio. Joint State and Dynamics Estimation With High-Gain Observers and Gaussian Process Models. IEEE Control Systems Letters, 2021, 5 (5), pp.1627-1632. 10.1109/LCSYS.2020.3042412 . hal-03098157

\section{HAL Id: hal-03098157 \\ https://hal.science/hal-03098157}

Submitted on 5 Jan 2021

HAL is a multi-disciplinary open access archive for the deposit and dissemination of scientific research documents, whether they are published or not. The documents may come from teaching and research institutions in France or abroad, or from public or private research centers.
L'archive ouverte pluridisciplinaire HAL, est destinée au dépôt et à la diffusion de documents scientifiques de niveau recherche, publiés ou non, émanant des établissements d'enseignement et de recherche français ou étrangers, des laboratoires publics ou privés. 


\title{
Joint state and dynamics estimation with high-gain observers and Gaussian process models
}

\author{
Mona Buisson-Fenet ${ }^{1,2,3}$, Valery Morgenthaler ${ }^{2}$, Sebastian Trimpe ${ }^{3}$ Florent Di Meglio $^{1}$
}

\begin{abstract}
With the rising complexity of dynamical systems generating ever more data, learning dynamics models appears as a promising alternative to physics-based modeling. However, the data available from physical platforms may be noisy and not cover all state variables. Hence, it is necessary to jointly perform state and dynamics estimation. In this paper, we propose interconnecting a high-gain observer and a dynamics learning framework, specifically a Gaussian process state-space model. The observer provides state estimates, which serve as the data for training the dynamics model. The updated model, in turn, is used to improve the observer. Joint convergence of the observer and the dynamics model is proved for high enough gain, up to the measurement and process perturbations. Simultaneous dynamics learning and state estimation are demonstrated on simulations of a mass-spring-mass system.
\end{abstract}

Index Terms-Machine Learning, Nonlinear systems identification, Observers for nonlinear systems

\section{INTRODUCTION}

$\mathbf{W}$ ITH the recent advances in control engineering, more and more complex dynamical systems are being considered, for which it is often difficult to derive physics-based models. Because of the availability of experimental data, as well as novel algorithms to process it, learning dynamics models directly from that data is an attractive alternative. However, this experimental data often originates from sensor measurements on the physical system, which can be noisy and may not reflect all state variables. On the one hand, reconstituting the full state from noisy, partial measurements falls into the area of state estimation and observer design. For nonlinear systems, this remains a challenging task for which knowledge of the dynamics is usually required [1]. On the other hand, most existing approaches for learning dynamics models require knowing the full state [2]. Therefore, joint state estimation and dynamics learning is needed, a problem often referred to in the machine learning community as inference and learning [3].

The design of observers for nonlinear systems is a complex task for which various approaches have been investigated (see [1] for an overview). We focus on the fairly large class of systems that can be expressed in the so-called observable canonical form (see Sect. II). For these systems, one can design High-Gain Observers (HGOs), which rely on a triangular

\footnotetext{
${ }^{1}$ Centre Automatique et Systèmes, Mines ParisTech, PSL University, Paris, France, florent.dimeglio@mines-paristech.fr, mona.buissonemines-paristech. fr

${ }^{2}$ ANSYS Research Team, ANSYS France, Villeurbanne, France, valery.morgenthalereansys.com

${ }^{3}$ Institute for Data Science in Mechanical Engineering, RWTH Aachen University, Aachen, Germany, trimped dsme.rwth-aachen. de
}

structure with increasing gain power to compensate for the nonlinearity farther from the measurement. HGOs have been used for a wide variety of applications [4], [5]. In particular, they provide robustness to model uncertainty, as practical convergence can be proved for high enough gain, given only an upper bound on the nonlinearity [6].

Learning dynamics models is also an active research topic. In particular, Gaussian process (GP) state-space models are increasingly used [7], [8]. These nonparametric models exhibit many advantageous properties for learning dynamical systems: they are flexible, data-efficient, probabilistic, and can easily incorporate prior knowledge (see [9] for details). Thanks to their analytical formulation, GPs also allow for theoretical guarantees [10], [11], which is rarely the case for nonparametric machine learning frameworks.

The problem of joint inference and learning for GP statespace models is tackled in its most general form in [3] using variational inference, by modeling the latent states as extra hyperparameters of the GP. The expectation maximization (EM) algortihm is applied: in the first step, measurements are collected and the posterior distribution of the GP is computed. In the second step, all hyperparameters, including the pseudo inputs and outputs representing the evolution of the latent states, are optimized to maximize the data log likelihood. Improvements of this approach have been proposed, e.g., by using more sophisticated loss functions or incorporating additional structure [12], [13]. However, the optimization procedure remains high-dimensional and non-convex. This leads to a high computational burden and a risk of overfitting, which can make the models difficult to train. Furthermore, no theoretical guarantees are yet provided for such methods.

Recent works tackle this problem by combining observer design and data-driven dynamics learning with universal approximators. The model is learned with a neural network using smooth, continuous-time weight update laws [14], [15] or a basis expansion [16], then added to an observer built as a copy of the system plus linear output injection terms. Limited theoretical guarantees have been shown [14], [16], but joint convergence has only been proved if suitable gains can be found by solving a large set of linear matrix inequalities [15]. However, this yields an unusual neural network model for $f$ and an observer with a high number of parameters left to tune, limiting the practical use of the framework.

In this work, we combine the predictive power of machine learning with existing convergence results for state estimation. Our main contribution is the design of a framework for simultaneous state and dynamics estimation, by combining a HGO that estimates the full state from measurements and a 
GP model that learns the unknown nonlinearity. Convergence guarantees for both the observer and the dynamics model are provided; practical applicability is discussed and demonstrated on simulations. This builds upon the scheme proposed in [17], in which the nonlinearity is considered as a state with partially known dynamics in an extended HGO, and is learned by an identifier satisfying certain requirements. The key difference of our approach is to directly learn a discrete model of the nonlinearity instead of differentiating it and extending the observer. This enables us to deal with controlled systems and input-dependent nonlinearities, and to decrease the error in the data used for regression. Furthermore, it decreases the dimensionality of the observer, which reduces noise amplification by the HGO. We also show that more flexible, non-parametric models such as GPs can learn the input-dependent dynamics while satisfying the smoothness assumptions which are necessary to prove joint convergence.

After formalizing the problem, we present the proposed framework in Sect. II. In Sect. III we show joint convergence of both state and dynamics estimation, then demonstrate our approach on a numerical example in Sect. IV. Comparison to related work and limitations are discussed in Sect. V, before concluding in Sect. VI.

\section{Problem formulation ANd PROPOSEd FRAMEWORK}

We consider a dynamical system of state $x \in \mathbb{R}^{n}$, output $y \in \mathbb{R}$, and bounded control input $u \in \mathbb{R}^{m}$, where $n, m \in \mathbb{N}$. For ease of notation we focus on the single-output case, but all results extend to multiple outputs by concatenation. We assume the following observable canonical form

$$
\dot{x}=A x+B f(x, u)+D(u)+d, \quad y=C x+\epsilon,
$$

with $f$ an unknown nonlinearity acting on the $n^{\text {th }}$ state $x_{n}$, while the rest of the dynamics follows a chain of integrators:

$$
A=\left(\begin{array}{cc}
0_{n-1} & I_{n-1} \\
0 & 0_{n-1}^{\top}
\end{array}\right), \quad B=\left(\begin{array}{c}
0_{n-1} \\
1
\end{array}\right), \quad C=\left(\begin{array}{c}
1 \\
0_{n-1}
\end{array}\right)^{\top} .
$$

The input function $D: \mathbb{R}^{m} \rightarrow \mathbb{R}^{n}$ is continuous and known while $d \in \mathbb{R}^{n}, \epsilon \in \mathbb{R}$ are unknown disturbances, typically considered deterministic (see Remark 2). All vectors are column vectors; $0_{n}$ denotes a vector of $n$ zeros, while $I_{n}$ is the identity matrix of size $n$. A broad class of systems can be transformed into this canonical form without knowing $f$, e.g., all differentially observable systems [1, Sect. 7.1]. Our aim is to compute an estimate $\hat{x}$ of the full state from measurements $y$, while jointly learning a model $\hat{f}$ of $f$. We make the following assumptions on (1).

Assumption 1: The true nonlinearity $f$ is Lipschitz continuous of constant $L_{f}$. There exist compact sets $\mathcal{X}$ and $\mathcal{U}$ such that $x(t) \in \mathcal{X}, u(t) \in \mathcal{U} \quad \forall t \geq 0$. Since $f$ is continuous on a compact space, its norm is also bounded by $f_{\max }$.

The proposed observer follows a cyclic structure, illustrated in Figure 1. During cycle number $j \in \mathbb{N}^{*}$, the observer produces an estimated state trajectory based on measurements and on the current dynamics model $\hat{f}_{j-1}$. This data is sampled and saved. At the end of the cycle, the model is updated based on the available estimated data. It produces an estimate $\hat{f}_{j}$, which is then used by the observer for the next cycle.

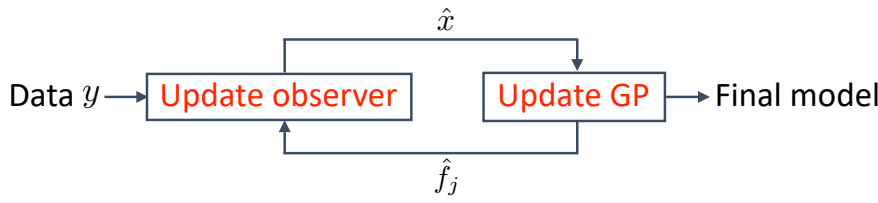

Fig. 1: Structure of the framework: after each cycle, an updated model $\hat{f}_{j}$ is computed and the observer is adapted.

\section{A. High-gain observer}

During cycle number $j$, the HGO performs state estimation using the current model of the dynamics $\hat{f}_{j-1}$ :

$$
\dot{\hat{x}}=A \hat{x}+B \hat{f}_{j-1}(\hat{x}, u)+D(u)+\Lambda(g)(y-C \hat{x}) .
$$

The gain is denoted $g>1$, while $\Lambda(g)$ is the gain matrix following a standard high-gain construction:

$$
\Lambda(g):=\left(\begin{array}{llll}
g L_{1} & g^{2} L_{1} & \cdots & g^{n} L_{n}
\end{array}\right)^{\top}
$$

with $L=\left(\begin{array}{lll}L_{1} & \cdots & L_{n}\end{array}\right)^{\top} \in \mathbb{R}^{n}$ such that $A-L C$ is Hurwitz. Equation (2) corresponds to the observer block in Figure 1. We make the following assumption, which is ensured by our dynamics model described hereafter.

Assumption 2: For all $j \in \mathbb{N}, \hat{f}_{j}$ is continuous and its norm is bounded by $\hat{f}_{\max }$.

Hence, we can pick $\mathcal{X}$ large enough such that $\hat{x}(t) \in \mathcal{X}$ $\forall t \geq 0$. With Assumptions 1-2, the error on the nonlinearity $\hat{f}(x, u)-f(x, u)$ is bounded. Then, as proved in [6] and used in the literature on HGOs, practical convergence in finite time can be shown in the absence of disturbances: for a given error level $\nu>0$ and a given time $\bar{t}>0$, there exists a gain $g$ high enough to ensure that for all $t \geq \bar{t},\|\hat{x}(t)-x(t)\| \leq \nu$. Hence, no matter how bad the approximation $\hat{f}$ of $f$ is, as long as an upper bound of the difference is known, practical convergence of the observer can be guaranteed for high enough gain. We leverage this property to build our method.

\section{B. Preliminaries on Gaussian processes}

In this paper we focus on Gaussian processes. However, any learning algorithm that satisfies our assumptions can be used. A Gaussian process (GP) is a collection of random variables, any finite subset of which is jointly normally distributed (see [9] for an overview). It is fully characterized by its mean function $m(\cdot)$ and its covariance function $k(\cdot, \cdot)$. Without loss of generality, we assume $m \equiv 0$. Any prior information can be included by substracting it from the output data in order to learn the residuals of this prior. Function properties such as smoothness are encoded in the choice of the kernel $k(\cdot, \cdot)$, which acts as a similarity measure for the values of $f(\cdot)$. At an unobserved point $x$, given a dataset $(X, Y)$ with Gaussian noise of variance $\sigma_{\epsilon}^{2}$ on the output, the GP prediction of $f(x)$ is normally distributed with posterior mean and variance

$$
\begin{aligned}
\mu(x \mid X, Y) & =\underline{k}(x)^{\top}\left(K+\sigma_{\epsilon}^{2} I\right)^{-1} Y \\
\sigma^{2}(x \mid X, Y) & =k(x, x)-\underline{k}(x)^{\top}\left(K+\sigma_{\epsilon}^{2} I\right)^{-1} \underline{k}(x),
\end{aligned}
$$

where $I$ is the identity matrix, $K=\left(k\left(x_{i}, x_{j}\right)\right)_{x_{i}, x_{j} \in X}$ is the covariance matrix of $X$, and $\underline{k}(x)=\left(k\left(x_{i}, x\right)\right)_{x_{i} \in X}$. The 
kernel $k$ usually depends on some hyperparameters, often obtained by maximizing the data marginal log likelihood.

Assumption 3: The kernel $k$ is differentiable, Lipschitz continuous of constant $L_{k}$, and its norm is bounded by $k_{\max }$. This is the case for most commonly used covariance functions, such as the squared exponential. GPs are increasingly used for learning dynamics thanks to their flexibility, data efficiency and analytical formulation. In this work, we use the GP posterior mean as a function approximator to estimate $f$.

\section{Learning method}

The state trajectory estimated by the observer is used to learn the dynamics model $\hat{f}$ through batch updates. For each update indexed by $j \in \mathbb{N}^{*}$, a dataset of length $N$ is constructed by sampling this trajectory with period $\Delta t$, starting from the last sample collected at $t_{j}$ :

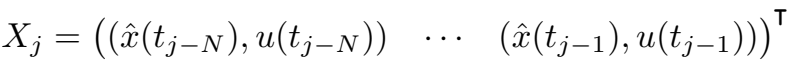

$$
\begin{aligned}
& Y_{j}=\left(\begin{array}{lll}
\hat{x}_{n}\left(t_{j-N+1}\right) & \cdots & \left.\hat{x}_{n}\left(t_{j}\right)\right)^{\top} .
\end{array}\right.
\end{aligned}
$$

Since the nonlinearity acts on the $n^{\text {th }}$ dimension, $x_{n}$ is the only output that needs to be collected. The $j^{\text {th }}$ update $\hat{f}_{j}$ is learned from inputs $X_{j}$ and outputs $Y_{j}$, then used in the observer (2) for the next cycle. It can be updated offline since this may necessitate more computing power, and the updates are not necessarily periodic. The model learned from (6) is

$$
\mu_{j}\left(\cdot \mid X_{j}, Y_{j}\right)=\underline{k}_{j}(\cdot)^{\top}\left(K_{j}+\sigma_{\epsilon}^{2} I_{N}\right)^{-1} Y_{j}
$$

with $K_{j}=\left(k\left(x_{i}, x_{l}\right)\right)_{x_{i}, x_{l} \in X_{j}}, \underline{k}_{j}(x)=\left(k\left(x_{i}, x\right)\right)_{x_{i} \in X_{J}}$. For the formulation of the GP posterior, Gaussian measurement noise of variance $\sigma_{\epsilon}^{2}$ is assumed. However, this need not be the case in reality as we only use the GP as a function approximator and $\sigma_{\epsilon}^{2}$, similarly to other hyperparameters, is chosen in practice for calibration purposes. No assumption on $\epsilon$ is needed for the provided theoretical guarantees.

We perform nonlinear regression to estimate the mapping $\mu$ : $(\hat{x}, u) \mapsto \hat{x}_{n}(t+\Delta t)$. However, $\hat{f}$ in the observer corresponds to the continuous time derivative of $\hat{x}_{n}$. Hence, we form $\hat{f}_{j}$ with a Euler differentiation step:

$$
\hat{f}_{j}(\hat{x}, u)=\frac{1}{\Delta t}\left(\mu_{j}\left(\hat{x}, u \mid X_{j}, Y_{j}\right)-\hat{x}_{n}\right) .
$$

To guarantee the boundedness of $\hat{f}$, we saturate it directly in the observer by imposing $\|\hat{f}\| \leq \hat{f}_{\max }$. The model (8) corresponds to the GP block in Figure 1; given the continuity of (7) and this saturation, it satisfies Assumption 2.

Remark 1: The choice of $\Delta t$ results from a trade-off: small enough to keep the numerical error from (8) low, large enough to see a real difference between two samples. Learning a discrete model from the estimated trajectory enables us to avoid extending the observer, contrarily to [17].

\section{Theoretical guaranteEs}

As stated previously, HGOs are robust to model uncertainty for systems in the observable canonical form. This enables us to decouple the procedures of state estimation and dynamics learning. Indeed, thanks to this robustness, convergence guarantees can still be obtained even in the worst-case scenario, i.e., with maximal but bounded model error, at the cost of a high gain. These convergence properties are then transferred to the dynamics model through its smoothness with respect to the dataset used for learning. Both practical and asymptotic convergence results are provided.

In the following proofs, we focus on the $\ell_{2}$ norm for vectors and matrices, but equivalent bounds can be obtained for any vector norm and its induced matrix norm. We first give a technical result on the smoothness of GP models, showing that the posterior mean is Lipschitz continuous not only with respect to the test point but also to the training dataset.

Lemma 1: Under Assumptions 1-3, the dynamics model $\hat{f}$ as defined in (8) is Lipschitz continuous with respect to each of its variables: $(x, u) \mapsto \hat{f}(x, u \mid X, Y)$ with constant $L_{x}$, and $(X, Y) \mapsto \hat{f}(x, u \mid X, Y)$ with constant $L_{z}$.

Sketch of proof: Assumptions 1-3 yield upper bounds on $k$ and $Y$. These and the Lipschitz continuity of $k$ directly yield the first claim. The sensitivity of the entries of $K$ with respect to each entry of $X$ is then bounded by elementary algebraic inequalities, and the second claim is obtained similarly.

Since the dataset $\left(X_{j}, Y_{j}\right)$ is constructed from state estimation samples, the error in this data directly depends on the state estimation error. This corresponds to the stability requirement in [17]. Then, Lemma 1 guarantees that any state estimation error in the dataset is smoothly transferred to the obtained model. This is essential to obtain stability guarantees, and corresponds to the regularity requirement in [17]. While we focus on GPs, any learning algorithm satisfying Lemma 1 based on a dataset constructed as in (6) can be used in our framework, to produce an observer and a dynamics model that can both be used for further control tasks.

\section{A. Practical convergence}

We denote $d_{g}=\left(\begin{array}{lll}g^{-1} d_{1} & \cdots & g^{-n} d_{n}\end{array}\right), \bar{x}=(x, u), \hat{\bar{x}}=$ $(\hat{x}, u)$ and $\|x\|_{\bar{t}}=\max \{\|x(t)\| \mid t \leq \bar{t}\}$. At a given $j \in \mathbb{N}^{*}$, $\hat{f}_{j}^{*}$ is the nonlinearity that would have been learned if the true data $X_{j}^{*}, Y_{j}^{*}$ had been available, i.e., if the state $x(t)$ had been directly available for sampling according to (6) instead of its estimate $\hat{x}(t)$. The prediction error is written as

$$
\varepsilon_{j}(\cdot)=\hat{f}_{j}(\cdot)-f(\cdot),
$$

while $\varepsilon_{j}^{*}(\cdot)=\hat{f}_{j}^{*}(\cdot)-f(\cdot)$ is the optimal prediction error that would have been obtained if the true state had been available instead of an estimate. We now state a first result on the practical convergence of the proposed framework.

Theorem 1: For system (1)-(2) with (8) under Assumptions $1-3$, for any given error level $\nu>0$, any time $\bar{t}>0$, there exists a gain $g^{*}$ large enough such that for all $g \geq g^{*}, t \geq \bar{t}$ and $j \in \mathbb{N}^{*}$ such that $\bar{t} \leq t_{j} \leq t$, in the absence of disturbances $(d \equiv \epsilon \equiv 0)$, we have for any fixed $\bar{x} \in \mathcal{X} \times \mathcal{U}$ :

$$
\max \left\{\|\hat{x}(t)-x(t)\|,\left\|\hat{f}_{j}(\bar{x})-\hat{f}_{j}^{*}(\bar{x})\right\|\right\} \leq \nu .
$$

Proof: The proof follows three steps. During a given cycle indexed by some fixed $j \in \mathbb{N}^{*}$, with $t_{j} \geq t \geq t_{j-1}$, the model error can be bounded by the maximal error: $\left\|\hat{f}_{j-1}(\cdot)-f(\cdot)\right\| \leq$ $\hat{f}_{\text {max }}+f_{\text {max }}$. Using existing proofs of the practical convergence of HGOs under bounded model uncertainty [6], [17], we show 
that there exist constants $\rho_{1}, \rho_{2}, \rho_{3}>0$ and a gain $g^{*}>0$ such that for all $g \geq g^{*}, i \in\{1, \ldots, n\}$ :

$$
\begin{gathered}
\left\|\hat{x}_{i}(t)-x_{i}(t)\right\| \leq \max \left\{g^{i-1} \rho_{0} e^{-\rho_{1} g t}\|\hat{x}(0)-x(0)\|,\right. \\
\left.\rho_{2} g^{i-n-1}, \rho_{3} g^{i-1}\left\|\left(d_{g}, \epsilon\right)\right\|_{t}\right\}:=B_{i}(t) .
\end{gathered}
$$

The key then lies in bounding the error on $X_{j}$ and $Y_{j}$ :

$$
\begin{aligned}
\left\|X_{j}-X_{j}^{*}\right\| & \leq \sum_{l=j-N}^{j-1} \sum_{i=1}^{n}\left\|\hat{x}_{i}\left(t_{l}\right)-x_{i}\left(t_{l}\right)\right\|, \\
\left\|Y_{j}-Y_{j}^{*}\right\| & \leq \sum_{l=j-N+1}^{j}\left\|\hat{x}_{n}\left(t_{l}\right)-x_{n}\left(t_{l}\right)\right\| .
\end{aligned}
$$

Therefore, the error on the input and output datasets used to learn $\hat{f}_{j}$ decreases as the state estimation error decreases, with a delay of $N$ time steps corresponding to the time before earlier samples with a larger error are forgotten. Applying Lemma 1 then (12)-(13) and (11) yields for any $\bar{x} \in \mathcal{X} \times \mathcal{U}$ :

$$
\begin{gathered}
\left\|\hat{f}_{j}(\bar{x})-\hat{f}_{j}^{*}(\bar{x})\right\| \leq L_{z} N(n+1) \max \left\{\rho_{3} g^{n-1}\left\|\left(d_{g}, \epsilon\right)\right\|_{t_{j}},\right. \\
\left.\frac{\rho_{2}}{g}, g^{n-1} \rho_{0} e^{-\rho_{1} g t_{j-N}}\|\hat{x}(0)-x(0)\|\right\} .
\end{gathered}
$$

Combining (11) and (14) as such leads to a joint practical convergence result, with an additional term corresponding to the disturbances. In the absence of disturbances, i.e., with $d \equiv \epsilon \equiv 0$, this concludes the proof.

\section{B. Asymptotic convergence}

Theorem 1 shows that the practical convergence guarantees obtained for HGOs with bounded nonlinearity extend to the complete error system. Both the state estimation error and the error made by the dynamics model due to seeing only estimated instead of true data can be made arbitrarily small arbitrarily fast, up to the disturbances, at the cost of a high gain. We now present an asymptotic convergence result arising from the practical convergence of HGOs in the presence of model uncertainty, by bounding this uncertainty depending on the data, the test point, and the optimal $\varepsilon^{*}$.

Theorem 2: For system (1)-(2) with (8) under Assumptions $1-3$, there exist constants $c, c^{\prime}, c^{\prime \prime}>0$ and a gain $g^{*}>0$ such that $\forall g \geq g^{*}, \forall i \in\{1, \ldots, n\}$ and for any fixed test point $\bar{x} \in \mathcal{X} \times \mathcal{U}$, we have

$$
\begin{aligned}
& \limsup _{t \rightarrow \infty}\left\|\hat{x}_{i}(t)-x_{i}(t)\right\| \leq c \max \{ \\
& \left.\quad g^{i-n-1} \limsup _{t+j \rightarrow \infty}\left\|\varepsilon_{j}^{*}(x, u)\right\|, g^{i-1} \limsup _{t \rightarrow \infty}\left\|\left(d_{g}, \epsilon\right)\right\|\right\}, \\
& \limsup _{t+j \rightarrow \infty}\left\|\hat{f}_{j}(\bar{x})-\hat{f}_{j}^{*}(\bar{x})\right\| \leq \max \{ \\
& \left.c^{\prime} g^{-1} \limsup _{t+j \rightarrow \infty}\left\|\varepsilon_{j}^{*}(x, u)\right\|, c^{\prime \prime} g^{n-1} \limsup _{t \rightarrow \infty}\left\|\left(d_{g}, \epsilon\right)\right\|\right\} .
\end{aligned}
$$

Proof: First, using definition (9) and Lemma 1, we show that for any fixed $j \in \mathbb{N}^{*},(x, \hat{x}, u) \in \mathcal{X} \times \mathcal{X} \times \mathcal{U}$, there exists $c_{1}>0$ such that

$$
\begin{aligned}
& \left\|\hat{f}_{j-1}(\hat{x}, u)-f(x, u)\right\| \\
& \quad \leq\left\|\hat{f}_{j-1}(\hat{x}, u)-\hat{f}_{j-1}^{*}(x, u)\right\|+\left\|\varepsilon_{j-1}^{*}(x, u)\right\| \\
& \quad \leq c_{1}\left(\|\hat{x}-x\|+\left\|X_{j-1}-X_{j-1}^{*}\right\|+\left\|Y_{j-1}-Y_{j-1}^{*}\right\|\right. \\
& \left.\quad+\left\|\varepsilon_{j-1}^{*}(x, u)\right\|\right) .
\end{aligned}
$$

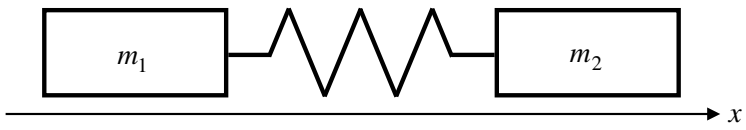

Fig. 2: Schematics of the mass-spring-mass system.

Then, using (17) in the proof of practical convergence of the HGO with $g$ large enough yields a bound similar to (11), but involving $\left\|X_{j-1}-X_{j-1}^{*}\right\|+\left\|Y_{j-1}-Y_{j-1}^{*}\right\|+\left\|\varepsilon_{j-1}^{*}(x, u)\right\|$ instead of the constant term in $\rho_{2}$. Going to the limit in both time and number of cycles and using (12)-(13) yields the first claim. Lemma 1 yields the second claim.

Theorem 2 bounds the difference between $\hat{f}$ and $\hat{f}^{*}$ that would have been obtained using true instead of estimated data for learning, without any assumption on the fit of the GP. If $\varepsilon^{*}$ is zero, both the state estimation and the prediction error are input-to-state stable with respect to the disturbances. If there are also no disturbances, they converge asymptotically. Since GPs with universal kernels are universal function approximators, $\varepsilon^{*}$ can get very small (it converges up to the numerical errors due to the Euler differentiation) as the number of samples grows to infinity, if the samples are densely distributed over $\mathcal{X} \times \mathcal{U}$, i.e., if the state-action space is well explored. However, if the hyperparameters of the GP do not enable a good fit or if the data is not rich enough, then $\varepsilon^{*}$ may be large. We further note that it is also possible to bound $\left\|\hat{f}_{j}(\bar{x})-f(\bar{x})\right\|$ similarly to (16) using the same error decomposition as (17): a term $\varepsilon_{j}^{*}(\bar{x})$ appears, representing the optimal error due to the GP model at the test point $\bar{x}$.

Remark 2: Nowhere do we use the form of $d$ and $\epsilon$. In principle, they could also be realizations of stochastic processes. However, Theorem 2 may then not be as meaningful as, depending on the process, limsup may not exist.

Remark 3: The proposed framework is modular: any regressor $\hat{f}$ can be used seamlessly instead of the GP. If Assumptions 1-2 and Lemma 1 are satisfied, the same theoretical guarantees will hold. This is the case for models of form $\hat{f}(\cdot)=\theta^{\top} \sigma(\cdot)$, where $\theta$ is a parameter to be learned from data following a Lipschitz procedure (such as recursive least squares), and $\sigma$ is a known, Lipschitz feature vector. Other observer designs providing guarantees similar to HGOs could also be considered, e.g., sliding mode observers [18].

Remark 4: In practice, one can learn the residuals model $\mu_{\text {res }}:(\hat{x}, u) \mapsto \hat{x}_{n}(t+\Delta t)-\hat{x}_{n}-\Delta t D_{n}(u)$ instead of $\mu$, and use $\mu(\hat{x}, u)=\mu_{\text {res }}(\hat{x}, u)+\hat{x}_{n}+\Delta t D_{n}(u)$ for prediction. This eases the training process by incorporating prior knowledge into the regression problem, while minimally changing the form of $Y_{j}$, which leads to a factor $(n+2)$ instead of $(n+1)$ in (14) and maintains the theoretical guarantees.

\section{Numerical SimULATION}

We demonstrate the performance of the proposed approach on a mass-spring-mass system with a nonlinear spring, as illustrated in Figure 2, and motivated by series-elastic actuator, e.g., [19]. We assume the system can be described by

$$
m_{1} \ddot{x}_{1}=f_{k}\left(x_{2}-x_{1}\right), \quad m_{2} \ddot{x}_{2}=-f_{k}\left(x_{2}-x_{1}\right)+u,
$$


where $x_{1}, x_{2}$ are the positions of the two objects, $m_{1}=m_{2}=1$ are their masses, and $f_{k}(\cdot)$ is some unknown nonlinear function representing the spring dynamics.

Assuming system (18) is differentially observable of order 4 (see [1] and references), it can be transformed into the observable canonical form (1). This is done by introducing a new state $z \in \mathbb{R}^{4}$, taking $z_{1}=x_{1}$ and computing the successive derivatives of $z_{1}$. As in (1), this yields

$$
\begin{gathered}
\dot{z}=A z+B f(z, u)+d \\
y=C z+\epsilon,
\end{gathered}
$$

where $f$ is an unknown nonlinearity. The proposed approach can directly be applied to (19) without further knowledge about $f$. In our simulations, we use $f_{k}(\cdot)=k_{1}(\cdot)+k_{2}(\cdot)^{3}$ with $k_{1}=0.3, k_{2}=0.1$ the spring constants. This yields for the true system in observable canonical form:

$$
\begin{aligned}
f(z, u)= & \frac{3 k_{2}}{m_{1} m_{2}}\left(u-\left(m_{1}+m_{2}\right) z_{3}\right) v_{1}^{2}+\frac{6 k_{2}}{m_{1}} v_{1} v_{2}^{2} \\
& +\frac{k_{1}}{m_{1} m_{2}}\left(u-\left(m_{1}+m_{2}\right) z_{3}\right), \\
\alpha= & \sqrt[3]{\frac{m_{1} z_{3}}{2 k_{2}}+\sqrt{\left(\frac{k_{1}}{3 k_{2}}\right)^{3}+\left(\frac{m_{1} z_{3}}{2 k_{2}}\right)^{2}}}, \\
\beta= & \sqrt[3]{\frac{m_{1} z_{3}}{2 k_{2}}-\sqrt{\left(\frac{k_{1}}{3 k_{2}}\right)^{3}+\left(\frac{m_{1} z_{3}}{2 k_{2}}\right)^{2}}}, \\
v_{1}= & \alpha+\beta, \quad v_{2}=\frac{z_{4}}{\frac{k_{1}}{m_{1}}+\frac{3 k_{2}}{m_{1}} v_{1}^{2}} .
\end{aligned}
$$

We simulate (19) with $d, \epsilon$ Gaussian noise of standard deviation $\sigma_{d}=\sigma_{\epsilon}=10^{-4}$ for ten cycles of 15 seconds each, sampled at $\Delta t=0.06 \mathrm{~s}$. We set $u(t)=0.4 \cos (1.2 t)$, $N=3000, g=10, L=(5,5,3,1)^{\top}$, and $\hat{f}_{0} \equiv 0$. We use a squared exponential kernel whose hyperparameters are fixed by maximizing the marginal log likelihood on a subset of data offline. We run 10 simulations from 10 initial conditions with $x_{1} \in[0,0.1], x_{2} \in[0.1,0.2]$, and $\dot{x_{1}}, \dot{x_{2}} \in[-0.005,0.005]^{1}$. For each, we start by precomputing a grid of random states and inputs, along with 50 test trajectories of 200 time steps, using a random initial state and one of three control strategies: random control, $u(t)=0.4 \cos (1.2 t)$, or $u(t)=0$.

In each simulation, we evaluate both the observer and the model at the end of every cycle. The observer (2) containing the current model is evaluated by computing the root mean square error (RMSE) between true and estimated trajectory over the last cycle, but also over the test trajectories, given $\hat{z}\left(t_{0}\right)=\left(y\left(t_{0}\right), 0,0,0\right)^{\top}$ and $y(t)$. The model (8) is evaluated by computing the RMSE of one step ahead predictions over the precomputed grid, and the RMSE of the predicted test trajectories, given the initial state but no measurements.

We observe joint convergence of the observer and the dynamics model. The error in all considered metrics decreases over time, as depicted in Figure 3. The numbers themselves are not necessarily meaningful, but their decreasing behavior and

\footnotetext{
${ }^{1}$ Code available at https://github.com/monabf/joint_state_ dynamics_estimation_HGOs_GPs.git
}

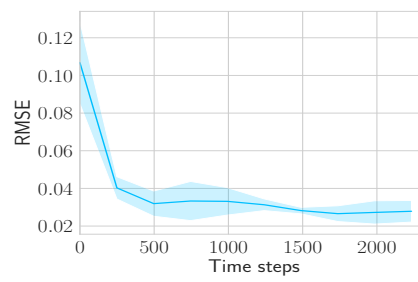

(a) State estimation over last cycle

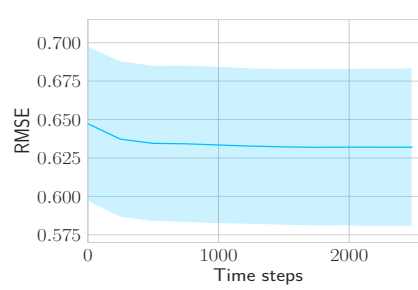

(c) Estimation of test trajectories

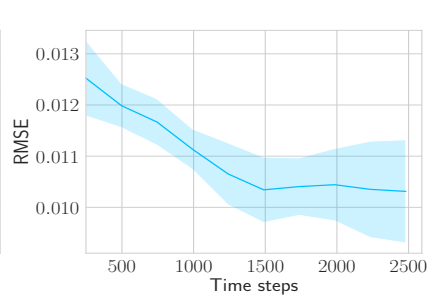

(b) GP predictions over a grid

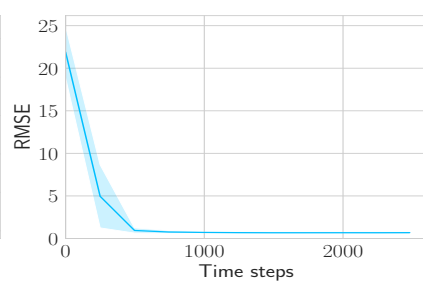

(d) Prediction of test trajectories
Fig. 3: RMSE of metrics over 10 simulations of (19) (mean \pm 2 standard deviations).

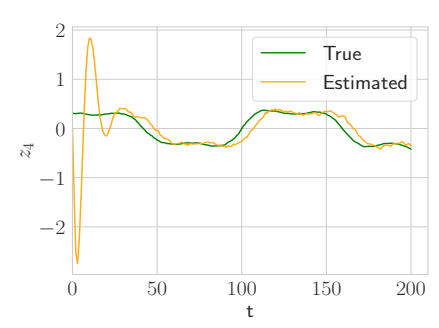

(a) Before learning

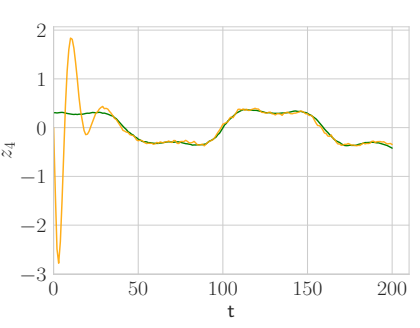

(b) After ten cycles
Fig. 4: Estimation of a test trajectory of $z_{4}$ (random control).

the visual results before and after a few cycles are significant. The variance is due to having different initial conditions, evaluation grids and test trajectories for each run rather than a different performance of the method. The remaining error is caused by the measurement and process noise, along with the irreducible model error given the available data. A test trajectory as estimated by the observer is presented in Figure 4 . Before the model is learned (a), the observer's estimates are delayed compared to the true state, because the observer has to wait for correction from the measurements. Once the model has been learned (b), the observer can anticipate and produce accurate estimates without delay. The phase portrait of another test trajectory predicted by the dynamics model is also depicted in Figure 5. It shows the final model can predict the first 100 time steps accurately, then slowly deviates.

Note that the same simulations were also run with the method proposed in [17]. By avoiding to differentiate $\hat{f}$, our method introduces less bias in the data used for regression, and the HGO has a lower dimensionality. As expected, the results seem to indicate that a discrete model learned directly from sampled trajectories yields a better dynamics model, which in turn yields a better observer. This difference grows with the dimensionality and the complexity of the considered nonlinearity.

\section{Discussion}

In this paper, we propose a framework for joint state and dynamics estimation of dynamical systems in the observable 


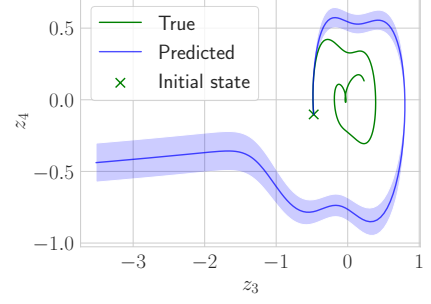

(a) After one cycle

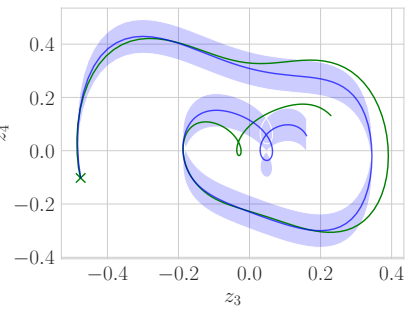

(b) After ten cycles
Fig. 5: Prediction of a test trajectory of $z_{4}$ against $z_{3}$ with sine control (mean \pm 2 standard deviations).

canonical form. Though the method shows promising results, it has some limitations that require further investigation. On the observer side, Theorems 1 and 2, like all theoretical guarantees provided by HGOs, only hold for high enough gain. However, using a large gain can be prohibitive in practice, mostly in high dimensions or with high measurement noise, as HGOs suffer from peaking and from noise amplification. This can be seen in Figure 4, where peaking is present and the measurement noise is already visible though it was rather low in the simulations $\left(\sigma_{\epsilon}=10^{-4}\right)$, making it difficult to deal with much higher noise. These effects can be mitigated by changing the gain [20] or using multiple HGOs [5], [21]. In future work, these could be combined with our method to allow for higher noise levels. We are also limited by the observable canonical form: many systems can be transformed into this form without knowledge of the dynamics, as in Sect. IV, but the transformation back into the original coordinates remains unknown. In future work, we plan on extending our ideas to more general classes of systems, for example by replacing the HGO with another observer.

On the learning side, we assume $\hat{f}$ is Lipschitz continuous with respect to the training data. This is the case for GPs with fixed hyperparameters. However, as soon as a nonconvex optimization procedure is involved, e.g., for online hyperparameter tuning or for training a neural network instead of a GP, this assumption is not satisfied. In this case, Theorems 1 and 2 do not hold. In practice, such tools can often still be used while maintaining performance. Another limitation of GPs is the dimensionality: as the computation of the posterior scales at $O\left(N^{3}\right)$, the GP model can only deal with up to about $10^{4}$ data samples. The sliding window of length $N$ used in (6) deals with this issue but leads to the loss of possibly useful data. Other methods such as sparse GP approximations [22] can be more efficient in practice, but whether the theoretical guarantees extend is open.

\section{CONCLUSION AND OUTLOOK}

Due to the imperfect state data provided by most physical platforms, joint estimation of state and dynamics is at the core of dynamics model learning from experimental data. This is a challenging problem in general: few approaches exist, even fewer provide convergence guarantees. In this paper, we propose a framework for joint state estimation and dynamics learning of nonlinear systems in the observable canonical form. A high-gain observer estimates the state trajectory, which is used for learning the nonlinearity with a non-parametric
Gaussian process model. Practical and asymptotic convergence of both the state and dynamics estimation can be guaranteed, so that after convergence, the observer and the dynamics model can be used for further control tasks. Simultaneous model learning and improved state estimation are demonstrated on a numerical example. In future work, we plan on extending our approach to further ease application.

\section{REFERENCES}

[1] P. Bernard, "Observer Design for Nonlinear Systems," in Lecture Notes in Control and Information Sciences, vol. 479, Springer International Publishing, 2019.

[2] D. Nguyen-Tuong and J. Peters, "Model learning for robot control: A survey," Cognitive Processing, vol. 12, pp. 319-340, 2011.

[3] R. Turner, M. P. Deisenroth, and C. E. Rasmussen, "State-Space Inference and Learning with Gaussian Processes," Proceedings of the 13th International Conference on Artificial Intelligence and Statistics, pp. 868-875, 2010.

[4] A. M. Dabroom and H. K. Khalil, "Discrete-time implementation of high-gain observers for numerical differentiation," International Journal of Control, vol. 72, no. 17, pp. 1523-1537, 1999.

[5] D. Astolfi, L. Marconi, L. Praly, and A. R. Teel, "Low-power peaking-free high-gain observers," Automatica, vol. 98, pp. 169-179, 2018.

[6] A. Tornambé, "High-gain observers for non-linear systems," International Journal of Systems Science, vol. 23, no. 9, pp. 1475-1489, 1992.

[7] J. Kocijan, Modelling and Control of Dynamic Systems Using Gaussian Process Models. Springer, 2016.

[8] M. Buisson-Fenet, F. Solowjow, and S. Trimpe, "Actively Learning Gaussian Process Dynamics," in Proceedings of the 2nd Conference on Learning for Dynamics and Control, vol. 120, pp. 5-15, 2020.

[9] C. E. Rasmussen and C. K. Williams, Gaussian Processes for Machine Learning. MIT Press, 2006.

[10] J. Umlauft and S. Hirche, "Learning stochastically stable Gaussian process state-space models," IFAC Journal of Systems and Control, vol. 12, 2020

[11] A. Lederer, J. Umlauft, and S. Hirche, "Uniform Error Bounds for Gaussian Process Regression with Application to Safe Control," in Advances in Neural Information Processing Systems, pp. 657-667, 2019.

[12] A. Doerr, C. Daniel, M. Schiegg, D. Nguyen-Tuong, S. Schaal, M. Toussaint, and S. Trimpe, "Probabilistic recurrent state-space models," Proceedings of the 35th International Conference on Machine Learning, pp. 1280-1289, 2018

[13] S. Melchior, F. Berkenkamp, S. Curi, and A. Krause, "Structured Variational Inference in Unstable Gaussian Process State Space Models," in Proceedings of the 2nd Conference on Learning for Dynamics and Control, vol. 120, pp. 147-157, 2020.

[14] F. Abdollahi, H. A. Talebi, and R. V. Patel, "A stable neural network observer with application to flexible-joint manipulators," IEEE Transactions on Neural Networks, vol. 17, no. 1, pp. 118-129, 2006.

[15] A. Chakrabarty, A. Zemouche, R. Rajamani, and M. Benosman, "Robust Data-Driven Neuro-Adaptive Observers with Lipschitz Activation Functions," in Proceedings of the 58th IEEE Conference on Decision and Control, pp. 2862-2867, 2019.

[16] A. Chakrabarty and M. Benosman, "Safe Learning-based Observers for Unknown Nonlinear Systems using Bayesian Optimization," arXiv preprint arXiv:2005.05888, pp. 1-20, 2020.

[17] M. Bin and L. Marconi, "Model identification and adaptive state observation for a class of nonlinear systems," IEEE Transactions on Automatic Control, 2020.

[18] Y. Shtessel, C. Edwards, L. Fridman, and A. Levant, "Sliding Mode Control and Observation," in Control Engineering, Birkhäuser, New York, 2016.

[19] I. Thorson and D. Caldwell, "A nonlinear series elastic actuator for highly dynamic motions," IEEE International Conference on Intelligent Robots and Systems, pp. 390-394, 2011.

[20] J. H. Ahrens and H. K. Khalil, "High-Gain Observers in the Presence of Measurement Noise: A Switched-Gain Approach," in Proceedings of the 17th IFAC World Congress, vol. 41, pp. 7606-7611, 2008.

[21] K. Esfandiari and M. Shakarami, "Bank of High-Gain Observers in Output Feedback Control: Robustness Analysis Against Measurement Noise," IEEE Transactions on Systems, Man, and Cybernetics: Systems, pp. 1-12, 2019.

[22] J. Quiñonero-Candela and C. E. Rasmussen, "A unifying view of sparse approximate Gaussian process regression," Journal of Machine Learning Research, vol. 6, pp. 1939-1959, 2005. 


\section{IEEE COPYRIGHT FORM}

To ensure uniformity of treatment among all contributors, other forms may not be substituted for this form, nor may any wording of the form be changed. This form is intended for original material submitted to the IEEE and must accompany any such material in order to be published by the IEEE. Please read the form carefully and keep a copy for your files.

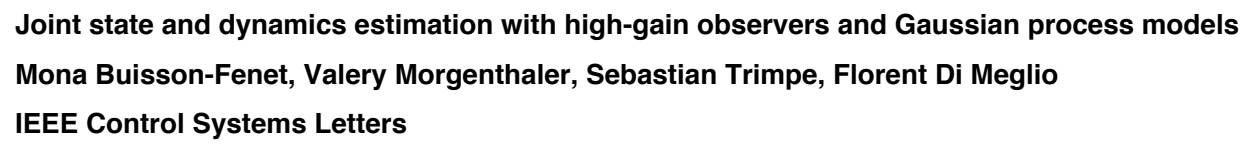

\section{COPYRIGHT TRANSFER}

The undersigned hereby assigns to The Institute of Electrical and Electronics Engineers, Incorporated (the "IEEE") all rights under copyright that may exist in and to: (a) the Work, including any revised or expanded derivative works submitted to the IEEE by the undersigned based on the Work; and (b) any associated written or multimedia components or other enhancements accompanying the Work.

\section{GENERAL TERMS}

1. The undersigned represents that he/she has the power and authority to make and execute this form.

2. The undersigned agrees to indemnify and hold harmless the IEEE from any damage or expense that may arise in the event of a breach of any of the warranties set forth above.

3. The undersigned agrees that publication with IEEE is subject to the policies and procedures of the IEEE PSPB Operations Manual.

4. In the event the above work is not accepted and published by the IEEE or is withdrawn by the author(s) before acceptance by the IEEE, the foregoing copyright transfer shall be null and void. In this case, IEEE will retain a copy of the manuscript for internal administrative/record-keeping purposes.

5. For jointly authored Works, all joint authors should sign, or one of the authors should sign as authorized agent for the others. 6. The author hereby warrants that the Work and Presentation (collectively, the "Materials") are original and that he/she is the author of the Materials. To the extent the Materials incorporate text passages, figures, data or other material from the works of others, the author has obtained any necessary permissions. Where necessary, the author has obtained all third party permissions and consents to grant the license above and has provided copies of such permissions and consents to IEEE

BY TYPING IN YOUR FULL NAME BELOW AND CLICKING THE SUBMIT BUTTON, YOU CERTIFY THAT SUCH ACTION CONSTITUTES YOUR ELECTRONIC SIGNATURE TO THIS FORM IN ACCORDANCE WITH UNITED STATES LAW, WHICH AUTHORIZES ELECTRONIC SIGNATURE BY AUTHENTICATED REQUEST FROM A USER OVER THE INTERNET AS A VALID SUBSTITUTE FOR A WRITTEN SIGNATURE.

Mona Buisson-Fenet

Signature
$30-11-2020$

Date (dd-mm-yyyy)

\section{Information for Authors}

\section{AUTHOR RESPONSIBILITIES}

The IEEE distributes its technical publications throughout the world and wants to ensure that the material submitted to its publications is properly available to the readership of those publications. Authors must ensure that their Work meets the requirements as stated in section 8.2.1 of the IEEE PSPB Operations Manual, including provisions covering originality, 
authorship, author responsibilities and author misconduct. More information on IEEE's publishing policies may be found at http://www.ieee.org/publications_standards/publications/rights/authorrightsresponsibilities.html Authors are advised especially of

IEEE PSPB Operations Manual section 8.2.1.B12: "It is the responsibility of the authors, not the IEEE, to determine whether disclosure of their material requires the prior consent of other parties and, if so, to obtain it." Authors are also advised of IEEE PSPB Operations Manual section 8.1.1B: "Statements and opinions given in work published by the IEEE are the expression of the authors."

\section{RETAINED RIGHTS/TERMS AND CONDITIONS}

- Authors/employers retain all proprietary rights in any process, procedure, or article of manufacture described in the Work.

- Authors/employers may reproduce or authorize others to reproduce the Work, material extracted verbatim from the Work, or derivative works for the author's personal use or for company use, provided that the source and the IEEE copyright notice are indicated, the copies are not used in any way that implies IEEE endorsement of a product or service of any employer, and the copies themselves are not offered for sale.

- Although authors are permitted to re-use all or portions of the Work in other works, this does not include granting third-party requests for reprinting, republishing, or other types of re-use.The IEEE Intellectual Property Rights office must handle all such third-party requests.

- Authors whose work was performed under a grant from a government funding agency are free to fulfill any deposit mandates from that funding agency.

\section{AUTHOR ONLINE USE}

- Personal Servers. Authors and/or their employers shall have the right to post the accepted version of IEEE-copyrighted articles on their own personal servers or the servers of their institutions or employers without permission from IEEE, provided that the posted version includes a prominently displayed IEEE copyright notice and, when published, a full citation to the original IEEE publication, including a link to the article abstract in IEEE Xplore. Authors shall not post the final, published versions of their papers.

- Classroom or Internal Training Use. An author is expressly permitted to post any portion of the accepted version of his/her own IEEE-copyrighted articles on the author's personal web site or the servers of the author's institution or company in connection with the author's teaching, training, or work responsibilities, provided that the appropriate copyright, credit, and reuse notices appear prominently with the posted material. Examples of permitted uses are lecture materials, course packs, ereserves, conference presentations, or in-house training courses.

- Electronic Preprints. Before submitting an article to an IEEE publication, authors frequently post their manuscripts to their own web site, their employer's site, or to another server that invites constructive comment from colleagues. Upon submission of an article to IEEE, an author is required to transfer copyright in the article to IEEE, and the author must update any previously posted version of the article with a prominently displayed IEEE copyright notice. Upon publication of an article by the IEEE, the author must replace any previously posted electronic versions of the article with either (1) the full citation to the IEEE work with a Digital Object Identifier (DOI) or link to the article abstract in IEEE Xplore, or (2) the accepted version only (not the IEEE-published version), including the IEEE copyright notice and full citation, with a link to the final, published article in IEEE Xplore.

Questions about the submission of the form or manuscript must be sent to the publication's editor.

Please direct all questions about IEEE copyright policy to:

IEEE Intellectual Property Rights Office, copyrights@ieee.org, +1-732-562-3966 